\title{
Pericarditis constrictiva: Causa de falla cardiaca en una paciente joven
}

\author{
Constrictive pericarditis: A cause of heart failure in a young patient \\ Jorge Quispe 1,a

\section{RESUMEN}

La pericarditis constrictiva es una causa de falla cardiaca derecha, que produce típicamente una alteración diastólica progresiva. Su corrección quirúrgica es posible con la pericardiectomía. La presentación en jóvenes y el compromiso importante de la función sistólica son escenarios infrecuentes. Se presenta el caso de una joven de 17 años con un cuadro de falla cardiaca congestiva. Los hallazgos de la ecocardiografía y resonancia cardiaca confirmaron el diagnóstico de pericarditis constrictiva. Se evidenció una disfunción sistólica con hipocinesia apical severa y con presencia de una masa a este nivel, compatible con trombo por la respuesta a anticoagulantes. La pericardiectomía se difirió por un alto riesgo operatorio y criterios de mal pronóstico por resonancia cardiaca.

PALABRAS CLAVE: Pericarditis constrictiva, falla cardiaca, trombo ventricular izquierdo. (Fuente: DeCS BIREME).

\section{SUMMARY}

Constrictive pericarditis is a cause of right heart failure that typically produces a progressive diastolic dysfunction. Pericardiectomy is the surgical indicated procedure. Presentation in young patients and systolic involvement are infrequent findings. We present the case of a 17 -year-old female patient with congestive heart failure; findings of the heart ultrasound and magnetic resonance confirmed the diagnosis of constrictive pericarditis. Systolic dysfunction with severe apical hypokinesis and a mass like lesion compatible with a thrombus were observed. Pericardiectomy was deferred for the high surgical risk and for the bad prognostic findings on the magnetic resonance.

KEYWORDS: Constrictive pericarditis, heart failure, left ventricular thrombus. (Source: MeSH NLM).

\footnotetext{
Servicio de Cardiologia, Hospital Nacional Cayetano Heredia. Lima, Perú.

a Médico Residente
} 


\section{INTRODUCCIÓN}

La falla cardiaca es un síndrome clínico debido de una alteración estructural o funcional del corazón. Afecta típicamente a adultos mayores; sin embargo, puede afectar también a jóvenes (1). Sus principales causas son la Cardiopatía isquémica e Hipertensiva, siendo las miocardiopatías primarias la principal causa en jóvenes. El compromiso pericárdico es una causa poco frecuente (2).

Las complicaciones asociadas a la falla cardiaca son de tipo hemodinámico, eléctrico, trombótico. El cardioembolismo es una complicación importante, representando la segunda causa después de la fibrilación auricular (3).

La pericarditis constrictiva es una entidad poco frecuente, producida por un engrosamiento fibrótico del pericardio que puede llegar a la calcificación, y que limita la distensibilidad del corazón. Se describe una alteración predominantemente diastólica (2). Su presentación clínica clásica corresponde al de una falla cardiaca derecha. Su etiología varía de acuerdo a la región; siendo en nuestro medio la etiología tuberculosa la más representativa (4).

La edad y forma de presentación, así como la complicación con trombo intracardiaco son infrecuentes, lo que motivó el reporte del caso.

\section{PRESENTACIÓN DEL CASO}

Mujer de 17 años, sin antecedentes patológicos de importancia, acudió al consultorio de neumología por tos y disnea de 3 meses de evolución. Se descartó tuberculosis activa, dado que tenía antecedente de contacto tuberculoso. La disnea progresó y se añadió
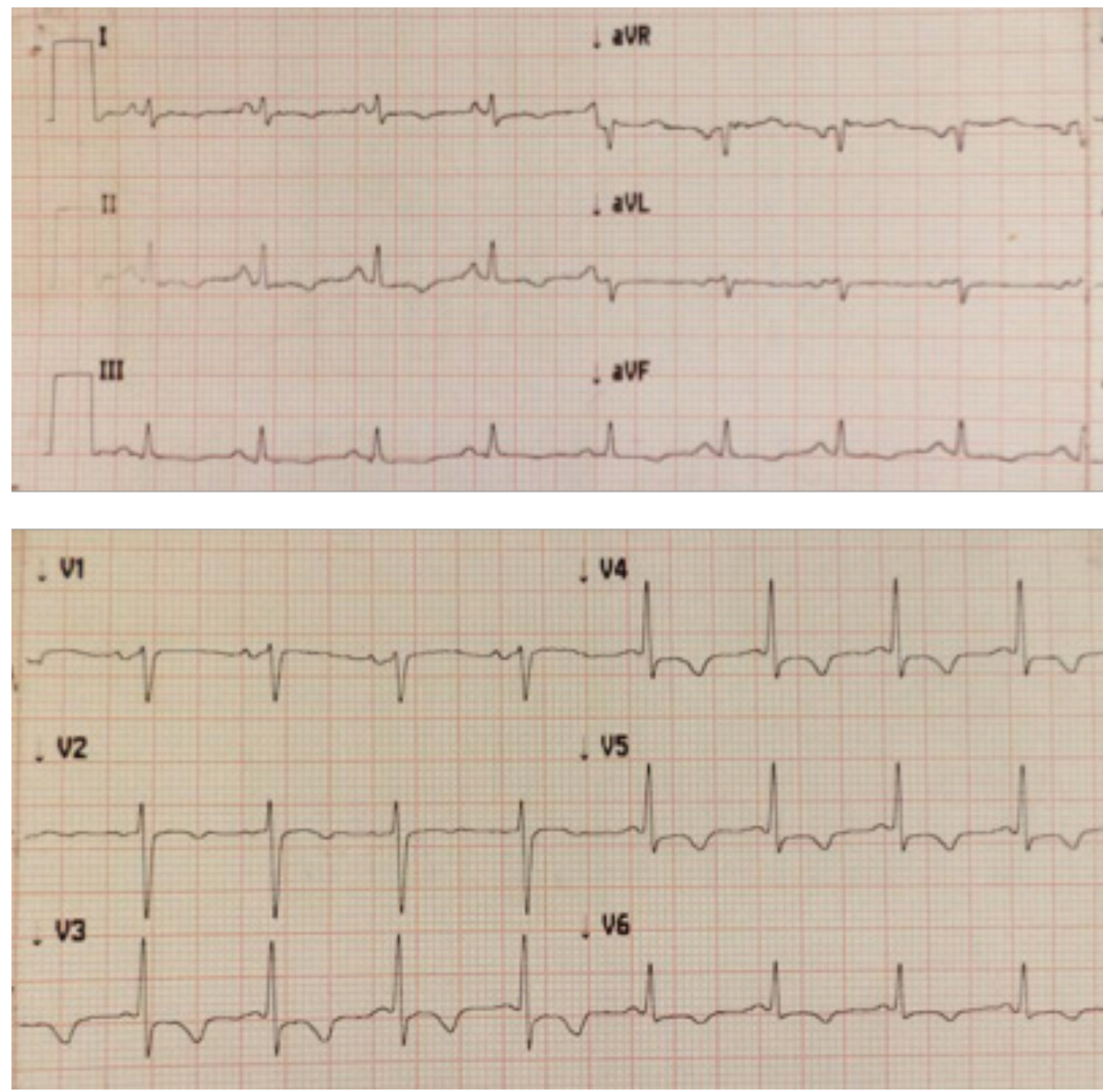

Figura 1.Electrocardiograma de 12 derivadas que muestra complejos de bajo voltaje en derivadas de las extremidades y trastorno difuso de la repolarización en derivadas precordiales. 
disnea paroxística nocturna, ortopnea y edema. La última semana presentó disnea al reposo por lo que fue hospitalizada.

El examen físico mostró funciones vitales normales. Al examen regional, se auscultaron crépitos en tercio inferior de ambos campos pulmonares, se evidenció edema que deja fóvea en miembros inferiores, ingurgitación yugular, reflujo hepatoyugular y hepatomegalia.

Los exámenes de laboratorio mostraron hemoglobina: 11,4 g/dl, leucocitos: $5510 \mathrm{cel} / \mathrm{mm}^{3}$ (neutrófilos: $80 \%$, linfocitos: 11\%), plaquetas 321 $000 \mathrm{cel} / \mathrm{mm}^{3}$, glucosa: $89 \mathrm{mg} / \mathrm{dl}$, creatinina: $0,6 \mathrm{mg} /$ dl, TGO: 39 U/1, TGP: $30 \mathrm{U} / 1$, TSH: 1,67 $\mu \mathrm{IU} / \mathrm{ml}$. El examen de orina no evidenció proteinuria y el sedimento fue normal. La baciloscopía en esputo fue negativa hasta en seis oportunidades. Los anticuerpos antinucleares fueron negativos.

El electrocardiograma de 12 derivadas reportó un ritmo sinusal, con complejos de bajo voltaje en derivadas de las extremidades y trastorno difuso de la repolarización, caracterizado por ondas $\mathrm{T}$ negativas difusas asimétricas en precordiales (Figura 1).

En la radiografía de tórax frontal se evidenció congestión leve, cardiomegalia y una tumoración en el tercio medio del campo pulmonar derecho (Figura
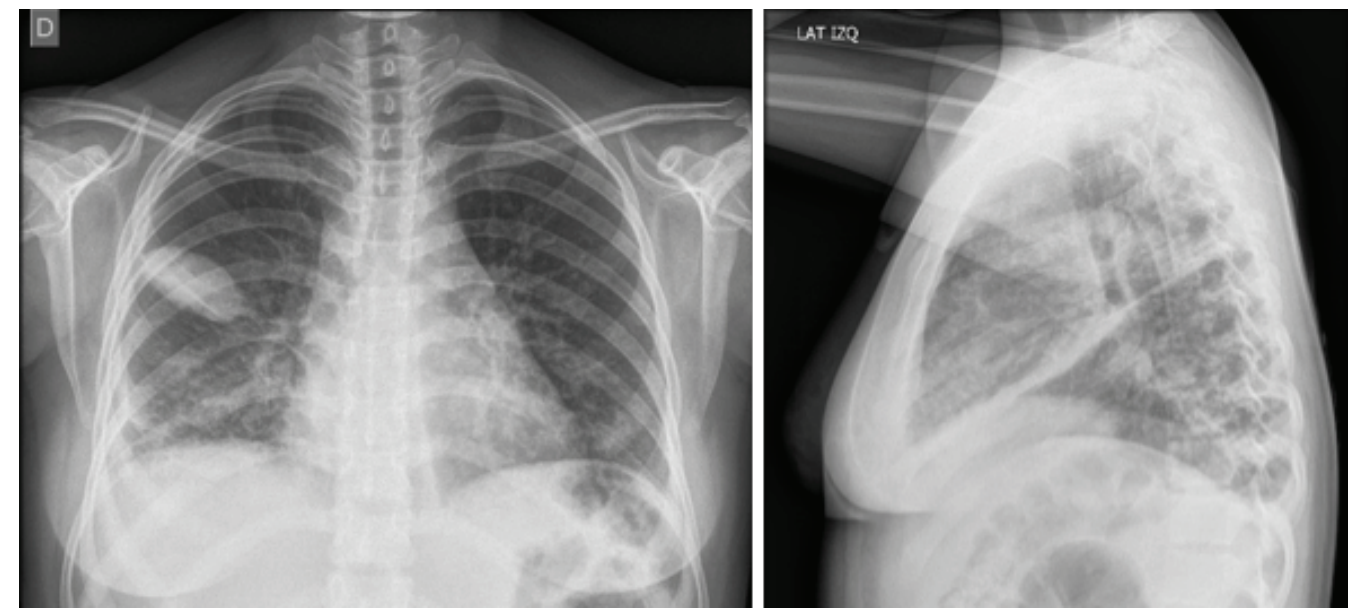

Figura 2. Radiografía de tórax (Póstero-anterior y lateral izquierda). Se evidencia cardiomegalia, redistribución del flujo pulmonar a predominio de bases pulmonares; así como, la presencia de un tumor fantasma a nivel de cisura horizontal derecha.

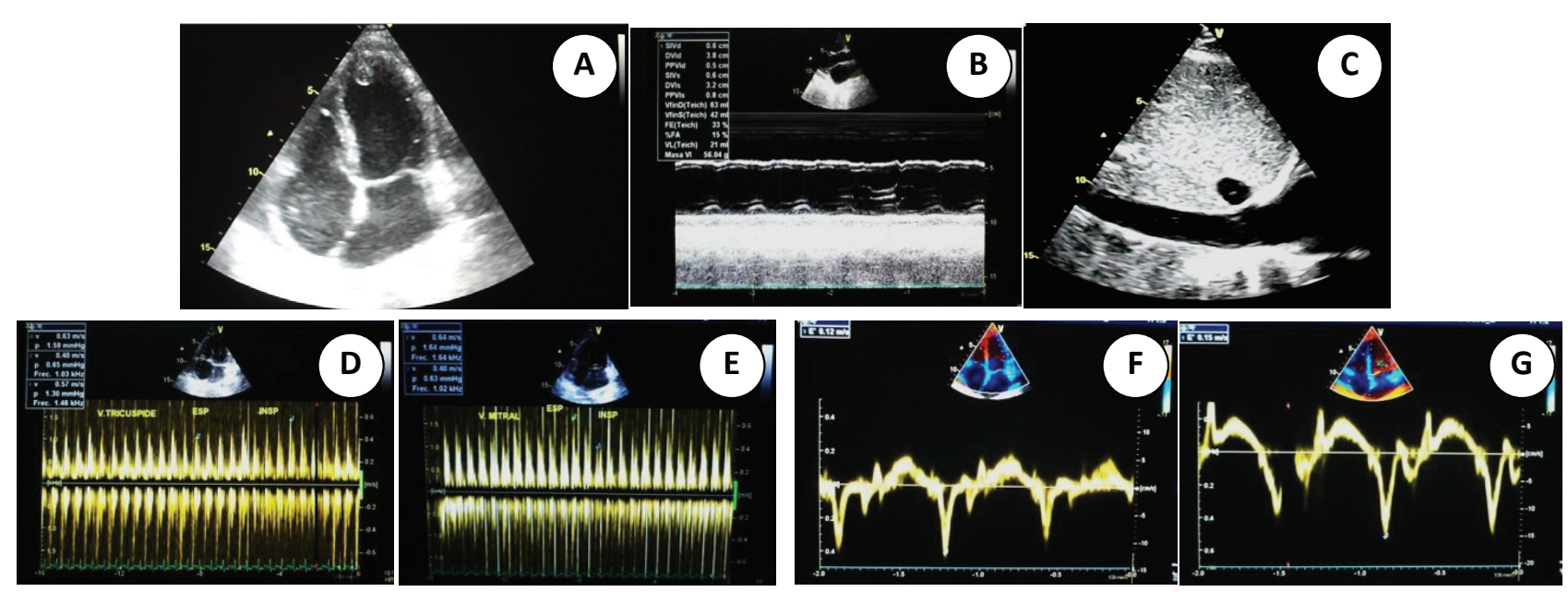

Figura 3. Estudio ecocardiográfico: A: Cuatro cámaras, donde se evidencia masa intracavitaria a nivel de ápice de ventrículo izquierdo. B: Modo $\mathrm{M}$ donde se ve notch protodiastólico en el septum interventricular. C: Dilatación de la vena cava inferior sin colapso inspiratorio. D y E: Variación respiratoria del flujo mitral y tricuspídeo mayor al 30\%. F y G: Doppler tisular lateral (F) y tisular (G) 


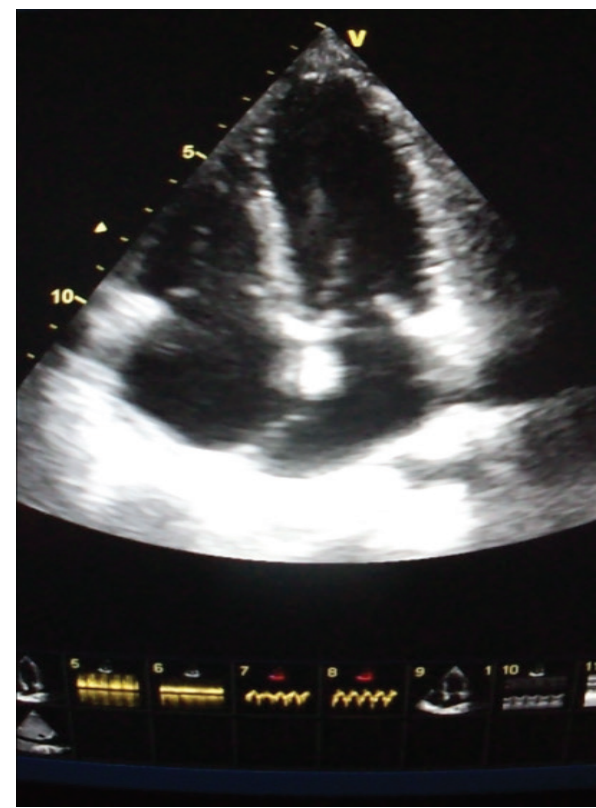

Figura 4. Ecocardiografía de control, donde se evidencia remisión del trombo intracavitario, luego de la terapia anticoagulante por dos semanas. Pero aún se observa contraste espontáneo en su interior.
2). En la proyección lateral izquierda, se evidenció que dicha tumoración correspondía a una colección de líquido a nivel de la cisura horizontal (Tumor fantasma).

La ecocardiografía reveló una dilatación biauricular leve. También se evidenció una disfunción sistólica moderada con fracción de eyección de 36\% con hipocinesia apical severa. Se encontró signos de fisiología constrictiva: movimiento oscilante del septum interventricular con la respiración, dilatación severa de la vena cava inferior sin colapso inspiratorio, variación respiratoria mayor al 30\% del flujo mitral y tricuspídeo, y annulus inversus en el Doppler tisular (Figura 3). Como hallazgo importante, se encontró una masa a nivel de ápice de ventrículo izquierdo, poco móvil, de bordes regulares asociado a contraste espontáneo, sugerente de trombo, que remitió con terapia anticoagulante (Figura 4). La resonancia cardiaca mostró un engrosamiento pericárdico heterogéneo a predominio apical y pared anterior. El "realce tardío" evidenció un componente inflamatorio importante (Figura 5).
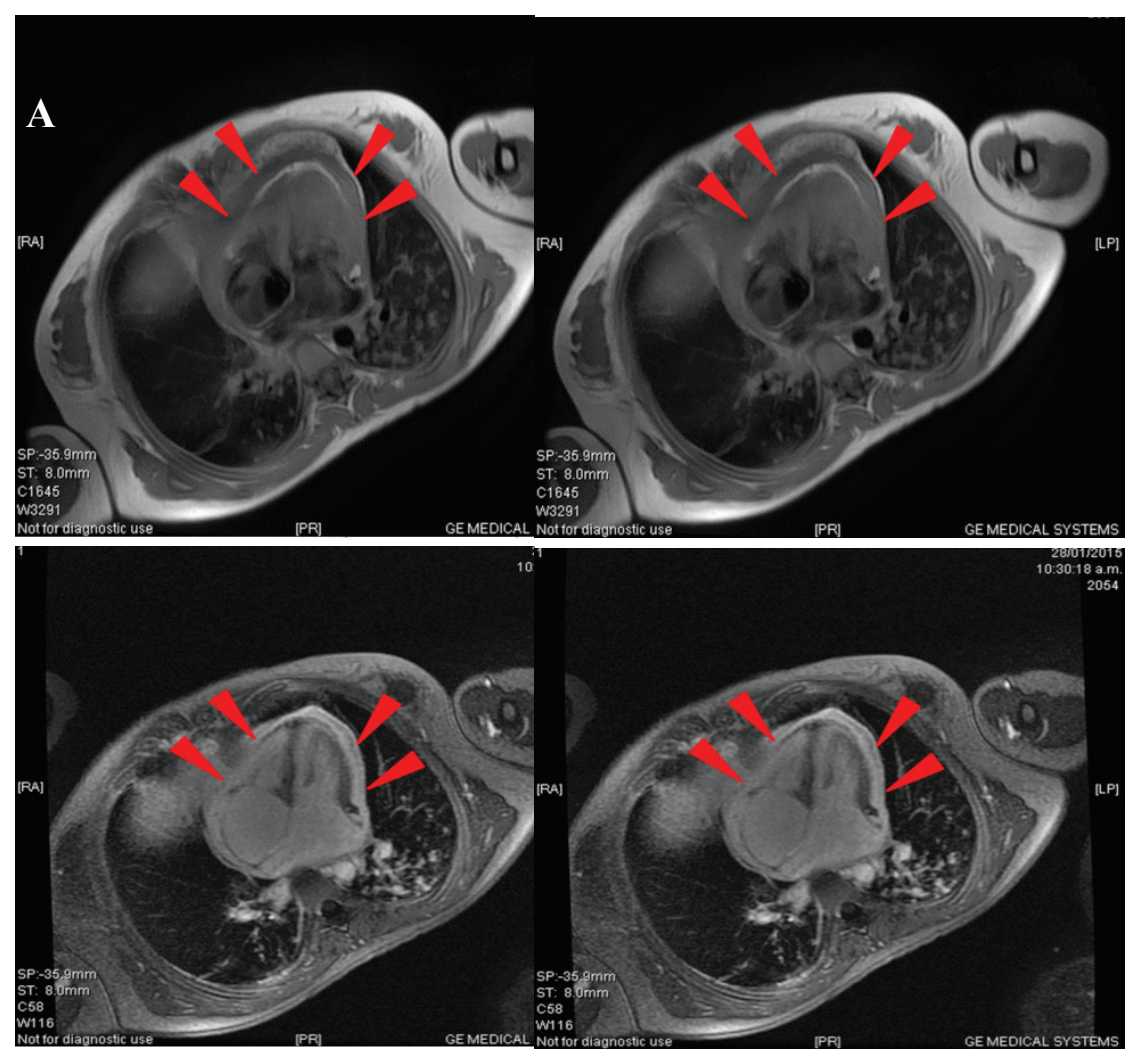

Figura 5. Imágenes de RMN cardiaca: A: Secuencia T1 eje largo, B: Secuencia T1 cuatro cámaras, C: contraste con realce tardío T1 eje largo, D: contraste con realce tardío T1 cuatro cámaras. Se evidencia un pericardio engrosado (Flechas) e con signos inflamatorios, de manera irregular y con compromiso de la hoja parietal y visceral, así como del miocardio subyacente. Asimismo, se evidencia el trombo intracavitario (Círculo en C). 
Se le inició tratamiento antituberculoso y corticoterapia a dosis altas. Así mismo, se le inició anticoagulación oral con Warfarina. En cuanto al tratamiento quirúrgico, la paciente presentaba alto riesgo operatorio. Según los parámetros actuales, la paciente cumplía criterios de "atrofia miocárdica", por lo que estaría contraindicada la cirugía. Se decidió valorar su respuesta al tratamiento médico y replantear la factibilidad del tratamiento quirúrgico.

En la evolución, se observó remisión de la masa intracavitaria en pocas semanas, con persistencia del cuadro congestivo.

\section{DISCUSIÓN}

Se reporta un caso de una joven con cuadro clínico compatible con falla cardiaca. Este grupo etario representa menos del $2 \%$ de la población con falla cardiaca (1). Llama la atención la presentación clínica, con un inicio de síntomas de compromiso izquierdo y en pocos meses un predominio clínico de cavidades derechas.

Las causas principales de falla cardiaca en jóvenes son la miocardiopatía primaria $(60 \%)$, seguida de la causa isquémica $(15 \%)(1)$. La causa más frecuente de falla derecha es la falla izquierda, pero generalmente este cuadro es más crónico (2). En el diagnóstico diferencial se encuentra la cardiopatía congénita y el cor pulmonar, siendo la pericarditis constrictiva una causa poco frecuente.

La pericarditis constrictiva es una complicación infrecuente de la pericarditis aguda (5). En países en vías de desarrollo, como el Perú, la causa tuberculosa representa una causa importante de esta entidad (4). Entre 30 a $60 \%$ de pacientes con pericarditis aguda tuberculosa desarrollan constricción, siendo el tiempo promedio 15 años (varias semanas hasta más de 20 años) (5). La fisiopatología se basa predominantemente, en una alteración de la función diastólica; sin embargo, existe evidencia de alteración sistólica a través del Strain ecocardiográfico en la motilidad de la pared antero-lateral del ventrículo izquierdo y del ventrículo derecho (6). Actualmente, la resonancia cardiaca juega un papel importante en su diagnóstico y en la orientación de la terapia médica (p.e. antiinflamatorios y realce tardío) (7), y quirúrgica.

En cuanto al tratamiento médico, la terapia antituberculosa coadyuvante para lograr remisión es controversial (8). En casos donde se demuestra un componente inflamatorio importante se sugiere la prueba terapéutica con antiinflamatorios por dos a tres meses.

La cirugía es la piedra angular del tratamiento. La pericardiectomía total o parcial mejora la fisiología cardiaca y la sintomatología; sin embargo, cuenta con un riesgo considerable (mortalidad peri operatoria de $6 \%$ en centros experimentados). Sin embargo existen situaciones clínicas (8) o estructurales que la contraindican, como la "atrofia miocárdica" (9). Se describe el término de "atrofia miocárdica", como un predictor de resultados adversos de la cirugía (9).

El manejo anticoagulante del trombo intracavitario con warfarina se vio generalmente en el contexto del síndrome coronario agudo (3). Teniendo en cuenta las dificultades asociadas a la warfarina; existen reportes satisfactorios con los nuevos anticoagulantes en este contexto (10).

\section{Declaración de financiamiento y de conflictos de intereses:}

El trabajo fue financiado por el autor. Declara no tener algún conflicto de intereses.

\section{Correspondencia:}

Jorge Isaac Quispe Cárdenas Calle Manuel Villar N ${ }^{\circ} 572$ Piso 3

San Martin de Porres, Lima, Perú.

Teléfono: 51962277644

Correo: jorge.quispe@upch.pe

\section{REFERENCIAS BIBLIOGRÁFICAS}

1. Wong Ch, Hawkins N, Jhund P. Clinical characteristics and outcomes of young and very young adults with heart failure: The CHARM Programme. JACC. 2013; 62:1845-54.

2. Syed F, Schaff H, Oh J. Constrictive pericarditis: curable diastolic heart failure. Nat Rev Cardiol. 2014; 11(9):530-44.

3. Gurbel P, Tantry U. Antiplatelet and anticoagulant agents in heart failure current status and future perspectives. J Am Coll Cardiol HF. 2014; 2:1-14.

4. Rossell J, La Rosa A. Pericarditis tuberculosa. Rev Perú Cardiol. 1996; 22(1):816.

5. Imazio $M$, Brucato $A$, Maestroni $S$, et al. Risk of constrictive pericarditis after acute pericarditis. Circulation. 2011; 124:1270-1275.

6. Kusunose K, Dahiya A, Popović Z. Biventricular mechanics in constrictive pericarditis comparison 
with restrictive cardiomyopathy and impact of pericardiectomy. Circ Cardiovasc Imaging. 2013; 6:399-406.

7. Feng D, Glockner J, Kim K. Cardiac magnetic resonance imaging pericardial late gadolinium enhancement and elevated inflammatory markers can predict the reversibility of constrictive pericarditis after antiinflammatory therapy. Circulation. 2011; 124:1830-7.

8. Adler Y, Charron P, Imazio M, et al. 2015 ESC Guidelines for the diagnosis and management of pericardial diseases: The Task Force for the Diagnosis and Management of Pericardial Diseases of the European Society of Cardiology (ESC)Endorsed by: The European Association for Cardio-Thoracic Surgery (EACTS). Eur Heart J. 2015; 36(42):292164.
9. Rienmuller R, Gurgan M, Erdmann E. CT and MR evaluation of pericardial constriction:a new diagnostic and therapeutic concept. J Thorac Imaging. 1993; 8(2):108-21.

10. Delewi R, Zijlstra F, Piek J. Left ventricular thrombus formation after acute myocardial infarction. Heart. 2012; 98(23):1743-9.

Recibido: 04/05/2016

Aceptado: 08/03/2017 